\title{
An enigma in the genetic responses of plants to salt stresses
}

\begin{abstract}
Soil salinity is one of the main factors restricting crop production throughout the world. Various salt tolerance traits and the genes controlling these traits are responsible for coping with salinity stress in plants. These coping mechanisms include osmotic tolerance, ion exclusion, and tissue tolerance. Plants exposed to salinity stress sense the stress conditions, convey specific stimuli signals, and initiate responses against stress through the activation of tolerance mechanisms that include multiple genes and pathways. Advances in our understanding of the genetic responses of plants to salinity and their connections with yield improvement are essential for attaining sustainable agriculture. Although a wide range of studies have been conducted that demonstrate genetic variations in response to salinity stress, numerous questions need to be answered to fully understand plant tolerance to salt stress. This chapter provides an overview of previous studies on the genetic control of salinity stress in plants, including signaling, tolerance mechanisms, and the genes, pathways, and epigenetic regulators necessary for plant salinity tolerance.
\end{abstract}

Keyword: Salinity stress; Osmotic tolerance; Tolerance mechanism and signaling pathways 